\title{
New Approach to Solve Fuzzy Linear Programming Problems by the Ranking Function
}

\author{
A. Karpagam and Dr.P. Sumathi
}

\begin{abstract}
In this paper, a new method is proposed to find the fuzzy optimal solution of fully fuzzy linear programming problems with triangular fuzzy numbers. A computational method for solving fully fuzzy linear programming problems (FFLPP) is proposed, based upon the new Ranking function. The proposed method is very easy to understand and to apply for fully fuzzy linear programming problems occurring in real life situations as compared to the existing methods. To illustrate the proposed method numerical examples are solved.
\end{abstract}

Keywords--- Fully Fuzzy Linear Programming Problem, Fuzzy Number, Fuzzy Variable Linear Programming, Fuzzy Optimal Solution, Ranking Function, Triangular Fuzzy Number

\section{INTRODUCTION}

$\mathrm{T}$ HE concept of Fuzzy logic was first conceived by Loft Zadeh, a professor at the university of California at Berkley. But as a way of processing data by allowing partial set membership rather than crisp set membership or nonmembership.Allahviranloo et al., (2008) [1] proposed a new method for solving fully fuzzy linear programming problems by the use of ranking function. Kumar et al.,(2010) [2] proposed a new method for solving fully fuzzy linear programming problems with inequality constraints. Amit Kumar \& Jagdeep Kaur,(2013) [3] proposed a new method for finding the fuzzy optimal solution of fully fuzzy linear programming problems with equality constraints. Bellman \& Zadeh, (1970) [4] proposed the concept of decision making in fuzzy environment. Cadenas \& Verdegay, (1997) [5] studied a linear programming problem in which all its elements are defined as fuzzy sets. (Chanas, 1983) [6] Proposed the possibility of the identification of a complete fuzzy decision in fuzzy linear Programming by use of the parametric programming technique. Fang et al., (1999) [7] presented a method for solving Linear programming problems with fuzzy coefficients in constraints. Jimenez et al.,(2007) [8] proposed a method for solving linear programming problems where all the coefficients are fuzzy numbers and used a fuzzy ranking method to rank the fuzzy objective values and to deal with the inequality relation on constraints. Kaur \& kumar (2012) [9]

A. Karpagam, Assistant Professor, Department of Mathematics, Valliammai Engineering College, Kattangulathur, Chennai, India. E-mail: karpagamohan@rediffmail.com

Dr.P. Sumathi, Research Scholar, Department of Mathematics, Easwari Engineering College, Ramapuram, Chennai - $600089 . \quad$ Email:psumathi16@rediffmail.com

DOI: 10.9756/BIJDM.6123 proposed a new method for solving exact fuzzy optimal solution of fully fuzzy linear programming problems with unrestricted fuzzy variables

Kolman \& Hill, (1984) [10] proposed a Fuzzy variable linear programming problems by use of a certain linear ranking function. Lotfi et al., (2009) [11] discussed fully fuzzy linear programming problems by representing all parameters and variables as triangular fuzzy numbers. Maleki et al.,(2000) [12] solved the linear programming problems in which all decision parameters are fuzzy numbers by the comparison of fuzzy numbers. Nagoor Gani \& Mohamed Assarudeen, (2013) [13] proposed a new operation on Triangular fuzzy number for solving fuzzy linear programming problem. Nasseri \&Alizadeh, (2011) [14] proposed a method for solving fuzzy linear programming problems by solving the classical linear programming. Nehi et al.,(2004) [15] defined the concept of optimality for linear programming problems with fuzzy parameters by transforming fuzzy linear programming problems into multiobjective linear programming problems. (Ramik,2005) [16]proposed the fuzzylinear programming problems based on fuzzy relations. (Werners,1987) [17] Introduced an interactive system which supports a decision maker in solving programming models with crisp or fuzzy constraints or fuzzy goals. Zhang et al., (2003) [18] proposed a method for solving fuzzy linear programming problems which involve fuzzy numbers in coefficients of objective functions. (Zimmerman,1978) [19] proposed the though fuzzy logic has been applied to many fields, from at control theory to artificial intelligence, it still remains controversial among most statisticians, who prefer Bayesian logic, and some control engineers, who prefer traditional two-valued logic.

Today, Fuzzy logic concept used widely in many implementations like automobile engine and automatic gear control systems, air conditioners, automatic focus control, video enhancement in TV sets, washing machines, behaviorbased mobile robots, sorting and handling data, Information Systems, traffic control systems and so on.In this paper, some preliminaries are presented in section II. Section III describes the existing methods. The section IV illustrates the new approach with one numerical example. Comparison results are presented in section V. Section VI concludes the paper.

\section{PRELIMINARIES}

\section{Definition}

The characteristic function $\mu_{\mathrm{A}}$ of a crisp set A C X assigns a value either 0 or 1 to each member in X. This function can be generalized to a function $\mu_{\tilde{A}}$ such that the value assigned to the element of the universal set $\mathrm{X}$ fall within a specified range 
i.e. $\mu_{\tilde{A}}: \mathrm{X} \rightarrow[0,1]$. The assigned value indicate the membership function and the set $\widetilde{A}=\left\{\left(\mathrm{x}, \mu_{\tilde{A}}(x)\right) ; \mathrm{x} \in \mathrm{X}\right\}$ defined by $\mu_{\tilde{A}}(x)$ for $\mathrm{x} \epsilon \mathrm{X}$ is called fuzzy set.

\section{Definition}

A fuzzy number $\tilde{A}=(\mathrm{m}, \alpha, \beta)$ is said to be triangular fuzzy number if its membership function is given by

$\mu_{\tilde{A}}(x)=\left\{\begin{array}{c}\frac{\frac{m-x}{\alpha}, \mathrm{m}-\alpha \leq \mathrm{x}<m, \quad}{1-\frac{x-m}{\beta}, \mathrm{m} \leq \mathrm{x}<m+\beta, \beta>0} \\ 0, \quad \text { otherwise }\end{array}\right.$

Definition

A fuzzy number $\tilde{A}=(\mathrm{m}, \alpha, \alpha)$ is said to be a symmetric triangular fuzzy number if its membership function is given by

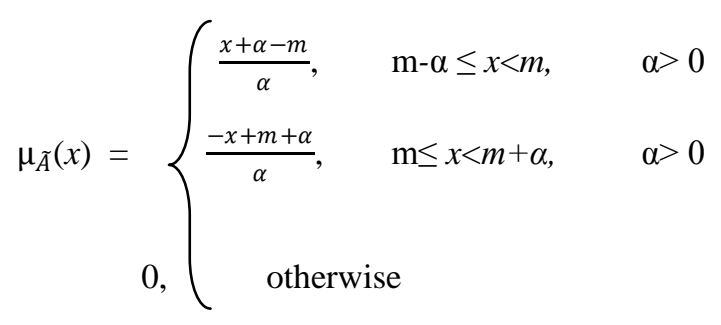

Definition

The concept of Fuzzy decision making was first proposed by Bellman \& Zadeh, (1970) [4].A linear programming problem is called fuzzy variable linear programming problem(FVLPP), if some of the parameters are crisp, and variables and right hand sides are fuzzy numbers. General form of FVLPP as follows:

$$
\begin{aligned}
& \text { Max (min) } \tilde{z}=c_{j} \tilde{x}_{j} \\
& \text { Subject to } A_{i j} \tilde{x}_{j} \leq \tilde{B}_{i} \\
& \tilde{x}_{j} \geq 0 \quad \text { Where } c_{j} \varepsilon R^{n}, A_{i j} \varepsilon(\mathrm{R})^{\mathrm{m} \times \mathrm{n}}, \tilde{B}_{i} \varepsilon(\mathrm{F}(\mathrm{R}))^{\mathrm{m}} \tilde{x}_{j} \varepsilon(\mathrm{F}(\mathrm{R}))^{\mathrm{n}}
\end{aligned}
$$

\section{Definition}

Kolman \& Hill,(1984) [10] was introduced a FFLP problem. A linear programming problem is called fully fuzzy linear programming problem (FFLPP), if parameters and variables are all fuzzy numbers. General form of FFLPP as follows:

$\operatorname{Max}(\min ) \tilde{z}=\tilde{c}_{j} \tilde{x}_{j}$

Subject to $\tilde{A}_{i j} \tilde{x}_{j} \leq \tilde{B}_{i}$,

$$
\tilde{x}_{j} \geq 0
$$

Where $\tilde{c}_{j} \varepsilon(\mathrm{F}(\mathrm{R}))^{\mathrm{n}}, \tilde{A}_{i j} \varepsilon(\mathrm{F}(\mathrm{R}))^{\mathrm{m} \times \mathrm{n}}, \tilde{B}_{i} \varepsilon(\mathrm{F}(\mathrm{R}))^{\mathrm{m}}, \tilde{x}_{j} \varepsilon(\mathrm{F}(\mathrm{R}))^{\mathrm{n}}$

\section{Definition}

An effective approach for ordering the elements of $F(R)$ is also to define a ranking function $\mathfrak{R}: \mathrm{F}(\mathrm{R}) \rightarrow \mathrm{R}$ which maps each fuzzy number into the real line, where a natural order exists. We define orders on $F(R)$ by: $\tilde{a} \geq \tilde{b} \quad$ if and only if $\mathrm{R}(\tilde{a}) \geq \mathrm{R}(\tilde{b})$

$\tilde{a}>\tilde{b} \quad$ if and only if $\mathrm{R}(\tilde{a}) \geq \mathrm{R}(\tilde{b})$

$\tilde{a}=\tilde{b} \quad$ if and only if $\mathrm{R}(\tilde{a})=\mathrm{R}(\tilde{b})$

\section{Definition}

Let $\tilde{A}=\left(a_{1}, b_{1}, c_{1}\right)$ and $\tilde{B}=\left(a_{2}, b_{2}, c_{2}\right)$ be two triangular fuzzy numbers, then

(i) $\tilde{A} \leq \tilde{B}$ iff $a_{1} \leq a_{2}, b_{1}-a_{1} \leq b_{2}-a_{2}, \mathrm{c}_{1}-b_{1} \leq \mathrm{c}_{2}-b_{2}$

(ii) $\tilde{A} \geq \tilde{B}$ iff $a_{1} \geq a_{2}, b_{1}-a_{1} \geq b_{2}-a_{2}, \mathrm{c}_{1}-b_{1} \geq \mathrm{c}_{2}-b_{2}$

(iii) $\tilde{A}=\tilde{B}$ iff $a_{1}=a_{2}, b_{1}=b_{2} \mathrm{c}_{1}=\mathrm{c}_{2}$

\section{EARLIER METHOD}

Amit kumar et.al., (2010) [2] has proposed to find the Fuzzy optimal solution of FFLP problems with inequality constraints by representing all the parameters are triangular fuzzy numbers.

\section{Maximize(Minimize) $(\widetilde{\boldsymbol{C}} \otimes \widetilde{\boldsymbol{x}})$ \\ Subject to $\widetilde{\boldsymbol{A}} \otimes \widetilde{\boldsymbol{x}} \leq,=, \geq, \widetilde{\boldsymbol{b}}$}

Where $\widetilde{\boldsymbol{x}}$ is a non-negative triangular fuzzy number and

$\widetilde{\boldsymbol{C}}=\left[\tilde{\boldsymbol{c}}_{j}\right]_{1 \times \mathrm{n}} \widetilde{\boldsymbol{A}}=\left[\widetilde{\boldsymbol{a}}_{i j}\right]_{\mathrm{m} \times \mathrm{n}} \widetilde{\boldsymbol{x}}=\left[\widetilde{\boldsymbol{x}}_{j}\right]_{\mathrm{n} \times 1} \widetilde{\boldsymbol{b}}=\left[\widetilde{\boldsymbol{b}}_{i}\right]_{\mathrm{m} \times 1}$

Example

$\operatorname{Max}(6,4,4) \tilde{\boldsymbol{x}}_{1}+(4,4,4) \tilde{\boldsymbol{x}}_{2}$

Subject to $(4,2,2) \widetilde{\boldsymbol{x}}_{1}+(2,2,2) \tilde{\boldsymbol{x}}_{2} \leq(10,6,6)$

$$
(6,6,6) \widetilde{\boldsymbol{x}}_{1}+(8,6,6) \widetilde{\boldsymbol{x}}_{2} \leq(8,2,2) \widetilde{\boldsymbol{x}}_{1}, \widetilde{\boldsymbol{x}}_{2} \geq 0
$$

Solution:

The standard form of given FFLPP

$$
\operatorname{Max}(6,4,4) \widetilde{\boldsymbol{x}}_{1}+(4,4,4) \widetilde{\boldsymbol{x}}_{2}
$$

Subject to $(4,2,2) \widetilde{\boldsymbol{x}}_{1}+(2,2,2) \widetilde{\boldsymbol{x}}_{2}+(1,1,1) \tilde{\boldsymbol{s}}_{1}=(10,6,6)$

$$
(6,6,6) \tilde{\boldsymbol{x}}_{1}+(8,6,6) \tilde{\boldsymbol{x}}_{2}+(1,1,1) \tilde{\boldsymbol{s}}_{2}=(8,2,2)
$$

$\widetilde{\boldsymbol{x}}_{1}, \widetilde{\boldsymbol{x}}_{2}, \widetilde{\boldsymbol{s}}_{1}, \widetilde{\boldsymbol{s}}_{2}$ are non-negative triangular fuzzy numbers

$\operatorname{Max}(6,4,4)\left(x_{1}, y_{1}, z_{1}\right)+(4,4,4)\left(x_{2}, y_{2}, z_{2}\right)$

Subject to

$(4,2,2)\left(x_{1}, y_{1}, z_{1}\right)+(2,2,2)\left(x_{2}, y_{2}, z_{2}\right)+(1,1,1)\left(s_{1}, t_{1}\right.$, $\left.u_{1}\right)=(10,6,6)$

$(6,6,6)\left(x_{1}, y_{1}, z_{1}\right)+(8,6,6)\left(x_{2}, y_{2}, z_{2}\right)+(1,1,1)\left(s_{2}, t_{2}\right.$, $\left.u_{2}\right)=(8,2,2)$

$\operatorname{Max} \mathfrak{R}\left(6 x_{1}+4 x_{2}, 4 y_{1}+4 y_{2}, 4 z_{1}+4 z_{2}\right)$

Subject to

$\left(4 x_{1}+2 x_{2}+s_{1}, 2 y_{1}+2 y_{2}+t_{1}, 2 z_{1}+2 z_{2}+u_{1}\right)=(10,6,6)$

$\left(6 x_{1}+8 x_{2}+s_{2}, 6 y_{1}+6 y_{2}+t_{2}, 6 z_{1}+6 z_{2}+u_{2}\right)=(8,2,2)$

$\left(x_{1}, y_{1}, z_{1}\right),\left(x_{2}, y_{2}, z_{2}\right),\left(s_{1}, t_{1}, u_{1}\right)\left(s_{2}, t_{2}, u_{2}\right)$ are nonnegative triangular fuzzy numbers. 
The above FFLPP is converted into the following Crisp linear programming:

$$
\text { Subject } \quad \begin{aligned}
& 4 x_{1}+2 x_{2}+s_{1}=10 \\
& 6 x_{1}+8 x_{2}+s_{2}=8 \\
& 2 y_{1}+2 y_{2}+t_{1}=6 \\
& 6 y_{1}+6 y_{2}+t_{2}=2 \\
& 2 z_{1}+2 z_{2}+u_{1}=6 \\
& 6 z_{1}+6 z_{2}+u_{2}=2 \\
& y_{1}-x_{1} \geq 0 \\
& y_{2}-x_{2} \geq 0
\end{aligned}
$$$$
\operatorname{Max}\left(\frac{\mathbf{1}}{\mathbf{4}}\left(12 x_{1}+8 x_{2}+4 y_{1}+4 y_{2}+4 z_{1}+4 z_{2}\right)\right)
$$

By simplex method, the Fuzzy optimal solutions are $\tilde{\mathrm{x}} 1=(1.33,0.33,0.33), \tilde{\mathrm{x}} 2=(0,0,0)$ and $\operatorname{Max} \tilde{\mathrm{z}}=10$.

\section{PROPOSED METHOD}

In this section, A new approach is proposed to find the Fuzzy optimal solution of fully fuzzy liner programming problems with symmetric triangular fuzzy numbers. The steps of the proposed method are as follows.

\section{Algorithm}

Step 1: Formulate the chosen problem into the following fully fuzzy linear programming problem:

$$
\operatorname{Max}(\min ) \tilde{\boldsymbol{z}}=\tilde{\boldsymbol{c}}_{\boldsymbol{j}} \tilde{\boldsymbol{x}}_{\boldsymbol{j}}
$$

Subject to $\widetilde{\boldsymbol{A}}_{\boldsymbol{i} j} \widetilde{\boldsymbol{x}}_{\boldsymbol{j}} \leq \widetilde{\boldsymbol{B}}_{\boldsymbol{i}}$,

$$
\widetilde{\boldsymbol{x}}_{\boldsymbol{j}} \geq 0
$$

Step 2: Using the Ranking function $\mathfrak{R}(\widetilde{\boldsymbol{C}}, \widetilde{\boldsymbol{A}})=\frac{\boldsymbol{m}+\mathbf{2} \boldsymbol{\alpha}-\boldsymbol{\beta}}{2}$ ,the FFLPP transform into FVLPP.
Step 3: Solve the FVLPP by using simplex method / Big$\mathrm{M}$ method. Let the solution be $\tilde{x}_{\mathrm{j}}$. Hence the solution of FFLPPis $\widetilde{x_{j}^{*}}$.

Step 4: Convert all the inequality constraints into equations by adding slack / surplus variable and the cost of this variable zero.

Step 5: Compute the value of $\tilde{Z}=\mathrm{C}_{\mathrm{B}} \mathrm{Y}_{\mathrm{j}}-\mathrm{C}_{\mathrm{j}} \mathrm{j} \neq \mathrm{B} \quad \mathrm{j}=1 \ldots . . \mathrm{n}$, If all $\tilde{Z} \geq 0 \forall j$ for maximization problem and $\widetilde{Z}<0 \forall j$ for minimization problem then the current solution is optimal, otherwise go to step 6 .

Step 6: Determine the basic variable $\tilde{x}_{\mathrm{k}}$, which will be replaced by the non-basic variable. Where $\mathrm{k}=$ argmini $\{\Re(\tilde{B})\}, \mathrm{i}=1,2 \ldots, \mathrm{m}$ in maximization problem and $\mathrm{k}=\operatorname{argmax}$ $\{\Re(\widetilde{B})\}, \mathrm{i}=1,2 \ldots, \mathrm{m}$ in minimization problem.

Step 7: Perform the pivot operation, return to step 5.

Example 4.1

$\operatorname{Max}(6,4,4) \tilde{x}_{1}+(4,4,4) \tilde{x}_{2}$

Subject to $(4,2,2) \tilde{x}_{1}+(2,2,2) \tilde{x}_{2} \leq(10,6,6)$

$$
(6,6,6) \tilde{x}_{1}+(8,6,6) \tilde{x}_{2} \leq(8,2,2)
$$

$\tilde{x}_{1}, \tilde{x}_{2} \geq 0$

Solution:

Standard form

$$
\begin{aligned}
& \operatorname{Max}(6,4,4) \tilde{x}_{1}+(4,4,4) \tilde{x}_{2}+0 \tilde{x}_{3}+0 \tilde{x}_{4} \\
& \text { Subject to }(4,2,2) \tilde{x}_{1}+(2,2,2) \tilde{x}_{2}+(1,1,1) \tilde{x}_{3}=(10,6,6) \\
& \quad(6,6,6) \tilde{x}_{1}+(8,6,6) \tilde{x}_{2}+(1,1,1) \tilde{x}_{4}=(8,2,2) \\
& \tilde{x}_{1}, \tilde{x}_{2}, \tilde{x}_{3}, \tilde{x}_{4} \geq 0
\end{aligned}
$$

Its equivalent FVLPP is

$\operatorname{Max} 5 \tilde{x}_{1}+4 \tilde{x}_{2}+0 \tilde{x}_{3}+0 \tilde{x}_{4}$

Subject to $3 \tilde{x}_{1}+2 \tilde{x}_{2}+\tilde{x}_{3}=(10,6,6)$

$$
6 \tilde{x}_{1}+5 \tilde{x}_{2}+\tilde{x}_{4}=(8,2,2)
$$

$\tilde{x}_{1}, \tilde{x}_{2}, \tilde{x}_{3}, \tilde{x}_{4} \geq 0$

Table 4.1.1

\begin{tabular}{|c|c|c|c|c|c|c|}
\hline Basis & $\tilde{x}_{1}$ & $\tilde{x}_{2}$ & $\tilde{x}_{3}$ & $\tilde{x}_{4}$ & RHS & $\Re(\tilde{B})=\frac{m+2 \alpha-\beta}{2}$ \\
\hline$\tilde{x}_{3}$ & 3 & 2 & 1 & 0 & $(10,6,6)$ & 8 \\
\hline$\tilde{x}_{4}$ & 6 & 5 & 0 & 1 & $(8,2,2)$ & 5 \\
\hline$\tilde{z}$ & -5 & -4 & 0 & 0 & $(0,0,0)$ & \\
\hline
\end{tabular}

Here -5 is the most negative in $\tilde{z}$, so $\tilde{x}_{1}$ is an entering variable and mini $\{\mathfrak{R}(10,6,6), \mathfrak{R}(8,2,2)\}$ is 5 . So $\tilde{x}_{4}$ is a leaving variable and its pivotal element is 6 .

Table: 4.1 .2

\begin{tabular}{|c|c|c|c|c|c|}
\hline Basis & $\tilde{x}_{1}$ & $\tilde{x}_{2}$ & $\tilde{x}_{3}$ & $\tilde{x}_{4}$ & RHS \\
\hline$\tilde{x}_{3}$ & 0 & $-\frac{3}{6}$ & 1 & $-\frac{3}{6}$ & $\left(\frac{36}{6}, \frac{30}{6}, \frac{30}{6}\right)$ \\
\hline$\tilde{x}_{1}$ & 1 & $\frac{5}{6}$ & 0 & $\frac{1}{6}$ & $\left(\frac{8}{6}, \frac{2}{6}, \frac{2}{6}\right)$ \\
\hline$\tilde{z}$ & 0 & $\frac{1}{6}$ & 0 & $\frac{5}{6}$ & $\left(\frac{40}{6}, \frac{10}{6}, \frac{10}{6}\right)$ \\
\hline
\end{tabular}


Since $\tilde{Z} \geq 0$, the fuzzy optimal solution of the FVLP problem is $\left.\tilde{x}_{1}=\left(\frac{8}{6}, \frac{2}{6}, \frac{2}{6}\right)\right), \tilde{x}_{2}=(0,0,0)$.Therefore, the fuzzy optimal solutions of FFLPP is $\widetilde{x_{1}^{*}}=\left(\frac{8}{6}, \frac{2}{6}, \frac{2}{6}\right), \widetilde{x_{2}^{*}}=(0,0,0)$ and the fuzzy optimal value is $\operatorname{Max} \tilde{z}=\left(\left(\frac{40}{6}, \frac{10}{6}, \frac{10}{6}\right)=10\right.$

\section{RESUlTS AND DisCUSSION}

The results of the fully fuzzy linear programming problem, chosen in the example, Obtained by using the existing method and proposed method are shown in Table 5.1 .

Table: 5.1

\begin{tabular}{|c|c|c|c|c|c|c|}
\hline \multirow{2}{*}{$\begin{array}{l}\text { Sl. } \\
\text { no }\end{array}$} & \multirow{2}{*}{$\begin{array}{l}\text { Number of } \\
\text { constraints }\end{array}$} & \multirow{2}{*}{$\begin{array}{c}\text { Number of } \\
\text { variables }\end{array}$} & \multicolumn{2}{|c|}{ Earlier method } & \multicolumn{2}{|c|}{ Proposed method } \\
\hline & & & $\begin{array}{l}\text { Number of } \\
\text { Iterations }\end{array}$ & $\begin{array}{l}\text { Fuzzy optimal value } \\
\tilde{z}\end{array}$ & $\begin{array}{l}\text { Number of } \\
\text { Iterations }\end{array}$ & $\begin{array}{l}\text { Fuzzy optimal } \\
\text { value } \widetilde{Z}\end{array}$ \\
\hline 1 & 2 & 2 & 9 & 10 & 2 & 10 \\
\hline 2 & 2 & 2 & 10 & 53 & 2 & 53 \\
\hline 3 & 2 & 2 & 10 & 62 & 2 & 62 \\
\hline 4 & 3 & 3 & 18 & 154 & 2 & 154 \\
\hline 5 & 3 & 3 & 14 & 216 & 2 & 216 \\
\hline 6 & 4 & 4 & 17 & 75 & 3 & 75 \\
\hline 7 & 4 & 4 & 16 & 112 & 3 & 112 \\
\hline 8 & 5 & 5 & 20 & 214 & 4 & 214 \\
\hline 9 & 5 & 5 & 19 & 198 & 6 & 198 \\
\hline 10 & 6 & 6 & 26 & 327 & 8 & 327 \\
\hline
\end{tabular}

It is obvious from the result: the existing method and proposed method the fuzzy optimal value are same.

\section{CONCLUSION}

In this paper a new method is proposed for solving the fuzzy optimal solution of FFLP problem transform into FVLP problems. The FFLP problem is converted into FVLP problem using new Ranking function. Ranking function is reasonable and effective for calculating the triangular weights of criteria. But the proposed method is less time consumption compared to previous method. Proposed method requires a less number of iterations. Therefore it is easier to solve fully fuzzy linear programming problem.

\section{REFERENCES}

[1] T. Allahviranloo, F. Hosseinzadeh, M.Kh Kiassary, N.A Kiani and L. Alizadeh, "Solving fully fuzzy linear programming problem by the ranking function”, Applied mathematical sciences, vol.8, pp. 19-32, 2008

[2] Amit kumar, Jagdeep kaur and pushpinder sign, "Fuzzy optimal solution of fully fuzzy linear programming problems with inequality constraints", International Journal of mathematical and computer science, pp. 37-40, 2010.

[3] Amit Kumar and Jagdeep Kaur, "General form of linear programming problems with fuzzy parameters”, Journal of Applied Research and Technology, vol.11, pp. 629-635, 2013.

[4] R.E Bellman and L.A Zadeh ,"Decision making in a fuzzy environment”, Management sciences, vol.17, pp. 141-164, 1970

[5] J.M. Cadenas and J.L. Verdegay, "Using Fuzzy Numbers in linear programming”, IEEE Transactions on Systems, vol. 27, pp. 10161022, 1997.

[6] S.Chanas, "The use of parametric programming in fuzzy linear programming”, Fuzzy Sets and Systems, vol. 11, pp. 229-241, 1983.

[7] S.C.Fang, C.F.Hu, H.F.Wang and S.Y. Wu, "Linear programming with fuzzy coefficients in constraints", Computers and Mathematics with Applications, vol.37, pp.63-76, 1999.

[8] M.Jimenez, M.Arenas, A.Bilbao and M.V. Rodrguez, "Linear programming with fuzzy parameters: An Interactive Method Resolution”, European Journal of Operation Research, vol. 177, pp. 1599-1609, 2007.
[9] J. Kaur and A. Kumar, "Exact fuzzy optimal solution of fully fuzzy linear programming problems with unrestricted fuzzy variables", Applied Intelligence, vol.37, pp. 145-154, 2012.

[10] Kolman and Hill, "Fuzzy variable linear programming by use of a certain linear ranking function”, Applied Mathematics, vol.18, 1984.

[11] F.H.Lotfi, T. Allahviranloo, M.A. Jondabeha and L.Alizadeh, "Solving a fully fuzzy linear programming using Lexicography method and fuzzy Approximate solution”, Applied Mathematical Modeling, vol.33, pp. 3151-3156, 2009

[12] H.R. Maleki, M.Tata and M. Mashinchi, "Linear programming with fuzzy variable”, Fuzzy sets and systems, pp. 21-33, 2000.

[13] A.Nagoor Gani and S.N. Mohamed Assarudeen, "A new operation on triangular fuzzy number for solving fully fuzzy linear programming problem", Applied Mathematical sciences, vol.6, No.11, pp. 525-632, 2012.

[14] S.H. Nasseri and Z.Alizade, "Solving linear programming problem fuzzy with right hand sides",Mathematics and computer science, vol.3, No.3, pp. 318-328, 2011.

[15] H.M.Nehi, H.R. Maleki and M.Mashinichi, "Solving Fuzzy number linear programming problem by Lexicographic Ranking function", Italian Journal of pure and applied Mathematics, vol .15, pp. 9-20, 2004.

[16] J.Ramik, "Duality in fuzzy linear programming: Some New concepts and Results", Fuzzy Optimization and decision Making, vol. 4, pp. 25-39, 2005

[17] B.Werners, "An interactive fuzzy programming system, Fuzzy sets and systems”, vol.23, pp. 131-147, 1987.

[18] G.Zhang, Y.H.Wu, M.Remias and J.Lu, "Formulation of Fuzzy linear programming problems as Four-Objective Constrained Optimization problems”, Applied Mathematics and Computation, vol. 139, pp. 383-399, 2003.

[19] H.J. Zimmermann, "Fuzzy programming and linear programming with objective functions", Fuzzy sets and systems, vol.1, pp. 37-46, 1978. 